\title{
Analysis on the Centroid Offset of Coupling Beam in Array-composite Resonator due to Process Variations
}

\author{
Haixia Yan, ${ }^{1}$ Jiaping Tao, ${ }^{2}$ and Linxi Dong ${ }^{2,3,4^{*}}$ \\ ${ }^{1}$ School of Information Engineering, Hangzhou Dianzi University, Hangzhou 310018, China \\ ${ }^{2}$ Key Laboratory of RF Circuits and System of Ministry of Education, College of Electronic and Information, \\ Hangzhou Dianzi University, Hangzhou 310018, China \\ ${ }^{3}$ State Key Laboratory of Functional Materials for Informatics, Chinese Academy of Sciences, \\ Shanghai 200050, China \\ ${ }^{4}$ State Key Laboratory for Manufacturing Systems Engineering, Xi'an Jiaotong University, Xi'an 710049, China
}

(Received May 2, 2018; accepted August 22, 2018)

Keywords: micro-electromechanical system (MEMS), micromechanical disk resonator, double-disk coupled resonator, centroid offset, PSpice simulation

Ideally, a mechanically coupled array-composite resonator can improve linearity and motional resistance by a factor equal to the number of constituent resonators used in the array. Owing to fabrication nonidealities, however, random variations in the dimensions of resonators and coupling beams often compromise the actual increase in output current. As a result, previous array composites have fallen short of their expected impedances and linearity improvements, particularly when the number of constituent resonators used in the array is large. Therefore, in this paper, we present a detailed theoretical analysis on the centroid offset of beams caused by process variations and some related substantive issues. Specifically, an equivalent circuit model for a coupling trapezoid beam is first deduced in the case of an offset centroid. Then, a model for a coupled double-disk resonator including a beam with an offset centroid is established by combining the equivalent circuit model with an existing circuit model of a radial-contour mode disk resonator. Finally, numerical results are obtained by simulations using ANSYS and PSpice with the mechanical and electrical models, respectively. It is shown that the resonant frequency varies by $45.7,110$, and $250 \mathrm{ppm}$ when the beam centroid shifts by 6,12 , and $18 \%$, respectively. When the centroid shifts towards one disk by $18 \%$, the output current amplitude of this disk decreases by $38.7 \%$ to $1.657 \mu \mathrm{A}$ (compared with $1.724 \mu \mathrm{A}$ at zero offset), while the output current amplitude of the other disk increases by $35.9 \%$ o to reach 1.785 $\mu \mathrm{A}$. In addition, when there is an offset, the $Q$ of the resonator decreases. In particular, when the centroid has an $18 \%$ offset, $Q$ decreases by nearly $19 \%$.

\section{Introduction}

The micromechanical resonators based on the capacitive transduction method are very attractive for various applications owing to their small size, on-chip integration potency with a CMOS circuit, and a high quality factor $(Q)$ at a very high frequency compared with those

*Corresponding author: e-mail: donglinxi@hdu.edu.cn https://doi.org/10.18494/SAM.2018.1971 
based on other transduction mechanisms. ${ }^{(1-4)}$ Although micro-electromechanical system (MEMS) resonators were reported to be used in the area of narrowband low-insertion-loss filters ${ }^{(5)}$ and low-power oscillators, ${ }^{(6)}$ there still exist some problems in micromechanical disk resonators: ${ }^{(7)} \mathrm{a}$ limited frequency range, a decrease in $Q$ with increasing operating frequency, and a very high motional resistance, which obstructs direct coupling or coupling using on-chip networks. As indicated in the literature, ${ }^{(3,8)}$ mechanically coupled array-composite resonators were also used to improve the motional resistance.

With this method, the linearity and motional resistance can be theoretically improved by a factor equal to the number of constituent resonators used in an array as shown in the literature. ${ }^{(3)}$ However, owing to fabrication nonidealities, random variations in the dimensions of resonators and coupling beams across the array often reduce the output current of the resonator array. As a consequence, previous array composites have fallen short of their expected impedance and linearity improvements, especially when the number of constituent resonators used in the array is large. Akgul et al. have conducted a voltage-controlled tuning method to optimize the output power of MEMS resonators. ${ }^{(9)}$ In particular, the problems caused by changes in disk radius and coupling beam length were studied, and a method of improving the overall performance of the resonator through electric stiffness tuning is proposed. Herein, we will focus on the problems of a centroid offset of coupling beams caused by process variations and explain its impact on the performance of array composite resonators with the main design parameters including resonance frequency, disk amplitude (or the amplitude of the output current), and $Q$.

The rest of this paper is organized as follows. The radial-contour mode disk resonator and its equivalent circuit model are introduced in Sect. 2, followed by some necessary formulae for later calculations. In Sect. 3, an ideal extensional-mode coupling beam and its equivalent circuit model are introduced. By further derivation, a beam model with cross-sectional area variations is established. Specifically, for simplification, in this study, a trapezoidal coupling beam model is adopted. Then, the equivalent circuit model of a double-disk coupled resonator is obtained in Sect. 4 using the equivalent circuit design method introduced by Wang and Nyugen. ${ }^{(10)}$ Finally, the numerical results from ANSYS and PSpice simulations are presented along with a discussion.

It is shown that the resonant frequency varies by $45.7,110$, and $250 \mathrm{ppm}$ when the beam centroid shifts by 6,12 , and $18 \%$, respectively. When the centroid shifts towards a disk, the output current amplitude of this disk decreases, while that of the other disk increases. All these changes are nearly linear versus the centroid offset. Typically, when the centroid shifts towards one disk by $18 \%$, the output current amplitude of this disk decreases by $38.7 \%$ o to $1.657 \mu \mathrm{A}$ (compared with $1.724 \mu \mathrm{A}$ at zero offset), while the output current amplitude of the other disk increases by $35.9 \%$ to reach $1.785 \mu \mathrm{A}$. In addition, when there is an offset, the $Q$ of the resonator decreases. In particular, when the centroid has an $18 \%$ offset, $Q$ decreases by nearly $19 \%$.

\section{Radial-contour Mode Disk Resonators}

In general, verification is accomplished through mechanical and electrical simulations. To perform these simulations, some consistent boundary conditions, which involve the conversions 
between the mechanical and electrical parameters, must be provided. In this section, radialcontour mode disk resonators are introduced and the boundary condition conversion method is given.

\subsection{Design and operation}

Figure 1 depicts a schematic perspective view of a disk resonator, with its key dimensions labeled, under a two-port bias and excitation scheme. As shown in Fig. 1, this device consists of a polysilicon disk suspended by a stem, aligned with its center, and enclosed by two polysilicon capacitive transducer electrodes with the gap $d_{0}$ (i.e., $87 \mathrm{~nm}$ ) from the disk circumference. Herein, it is assumed that the disk radius $R_{\text {disk }}$ is $18 \mu \mathrm{m}$, the thickness $t$ is $2.1 \mu \mathrm{m}$, and the stem has a diameter of $2 \mu \mathrm{m}$ and a height of $0.7 \mu \mathrm{m}$ from the substrate to the disk bottom.

To excite this disk resonator, a direct current (DC) bias voltage $V_{P}$ and an alternating current (AC) signal voltage $v_{i}$ are applied to the disk and input electrode, respectively. Note that the DC bias $V_{P}$ serves only to charge the electrode-to-disk capacitance and thereby does not incur power consumption. Together, these voltages generate an electrostatic input force $F_{i}$ in the radial direction (pointing outward from the disk) as follows: ${ }^{(11)}$

$$
F_{i}=\frac{1}{2}\left(\frac{\partial C_{1}}{\partial r}\right)\left(V_{p}-v_{i}\right)^{2} \cong-V_{p}\left(\frac{\partial C_{1}}{\partial r}\right) v_{i}
$$

where only the dominant term is retained at resonance (i.e., the components at DC and at frequencies different from $v_{i}$ are neglected) and $\partial C_{1} / \partial r$ is the change in electrode-to-resonator overlap capacitance per unit radial displacement at the input port (i.e., port 1).

Furthermore, $\partial C_{1} / \partial r$ can be approximated as follows: ${ }^{(12)}$

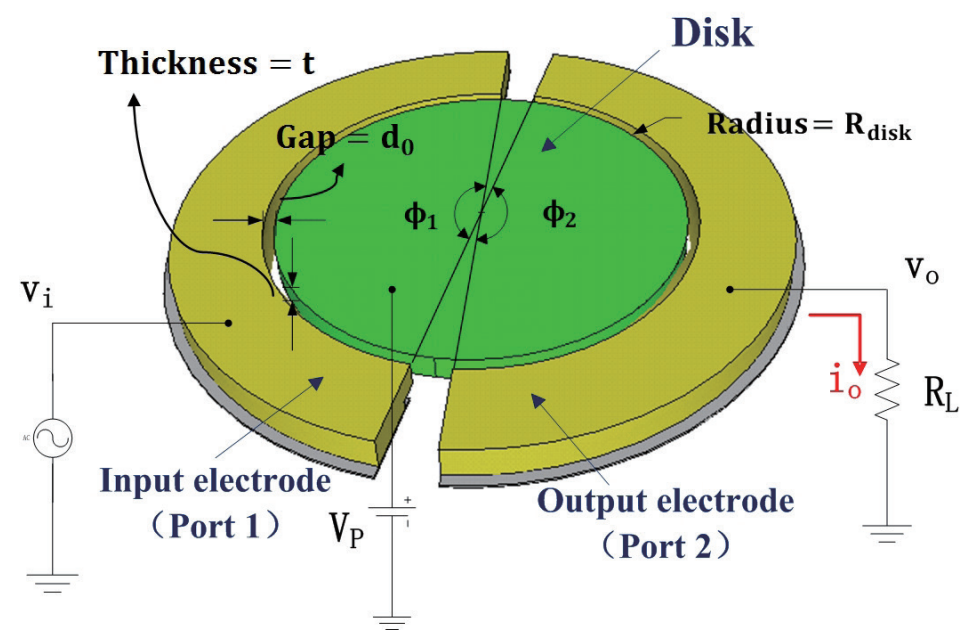

Fig. 1. (Color online) Schematic perspective view of a micromechanical disk resonator under a two-port bias and excitation scheme. 


$$
\frac{\partial C_{1}}{\partial r} \approx \frac{\varepsilon_{0} \phi_{1} R_{\text {disk }} t}{d_{0}^{2}}
$$

where $R_{\text {disk }}$ and $t$ are respectively the radius and thickness of the disk, $\varepsilon_{0}$ is the permittivity in vacuum, $d_{0}$ is the electrode-to-resonator gap spacing, and $\phi_{1}$ is the angle defined by the edges of the input electrode (i.e., electrode 1) as shown in Fig. 1.

To realize the loading of the drive force on the disk in the ANSYS simulation, the above electrostatic force is converted to pressure as

$$
P_{i}=\frac{F_{i}}{W_{e} \cdot t},
$$

where $W_{e}$ denotes the electrode width.

When the frequency of the $\mathrm{AC}$ signal voltage $v_{i}$ matches the radial-contour mode resonance frequency of the disk, the resulting force drives the disk into a vibration mode in which it expands and contracts radially around its circumference, as shown in Fig. 2. The radial displacement amplitudes at point $(r, \theta)$ on the disk and at the perimeter (i.e., $r=R_{\text {disk }}$ ) in phase form are respectively given by ${ }^{(13)}$

$$
\begin{aligned}
& \Re(r, \theta)=A h J_{1}(h r), \\
& \Re\left(R_{\text {disk }}, \theta\right)=\frac{Q F_{i}}{j k_{r e}},
\end{aligned}
$$

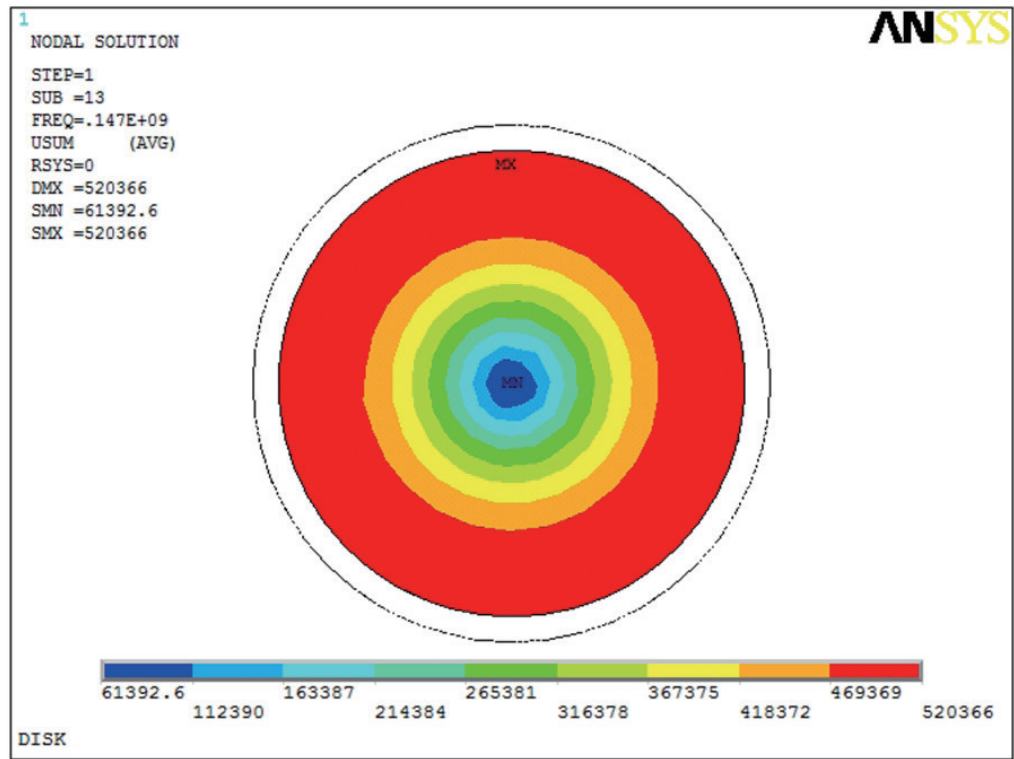

Fig. 2. (Color online) First-order mode shape of the radial-contour mode disk resonator. 
where $k_{r e}$ is the effective stiffness at the perimeter, $J_{n}(y)$ is the Bessel function of the first kind of order $n, A$ is the drive-force-dependent ratio ${ }^{(13)}$ (specified later in the tables in Sect. 5), and $h$ is a constant defined as

$$
h=\sqrt{\frac{\omega_{0}^{2} \rho}{\left(\frac{2 E}{2+2 \sigma}+\frac{E \sigma}{1-\sigma^{2}}\right)}},
$$

where $\omega_{0}=2 \pi f_{0}$ is the angular resonance frequency and $\rho, \sigma$, and $E$ are the density, Poisson ratio, and Young's modulus of the structural material, respectively.

The radial vibration of the disk creates a DC-biased time-varying capacitance between the disk and the output electrode, which generates an output motional current $i_{o}$ proportional to the amplitude of vibration as

$$
i_{o}=V_{p}\left(\frac{\partial C_{2}}{\partial r}\right) \frac{\partial \Re\left(R_{\text {disk }}, \theta\right)}{\partial t},
$$

where $\partial C_{2} / \partial r$ is the change in electrode-to-resonator overlap capacitance per unit radial displacement at the output port (i.e., port 2), which takes a form similar to that of Eq. (2) but with $\phi_{1}$ replaced by $\phi_{2}$. Note that, by using this formula, the ANSYS simulation results can be converted to the corresponding current values, which can be compared with the PSpice simulation results.

In summary, this disk resonator operates by first converting the input electrical signal $v_{i}$ to the mechanical force $F_{i}$, which is filtered by the high- $Q$ mechanical response of the resonator to pass only the components at the disk resonance frequency being converted to the disk displacement $\mathfrak{R}(r, \theta)$ [or velocity $v(r, \theta)$ ]. Then, this displacement is converted from the mechanical domain back to the electrical domain into the output current $i_{o}$ via the output electrode capacitive transducer. ${ }^{(14,15)}$

\subsection{Electrical equivalent circuits}

To perform the circuit simulation, it is necessary to convert the mechanical model of the disk resonator into an equivalent circuit model. Despite its mechanical nature, the disk resonator in Fig. 1 still has the appearance of an electrical device owing to its ports. It can be modeled by the electrical inductor-capacitor-resistor (LCR) equivalent circuits shown in Fig. 3. The element values in the LCR equivalents are governed by the total integrated kinetic energy in the resonator, its mode, and the parameters associated with its transducer ports. ${ }^{(11,14,16)}$

The equivalent mass at a location $(r, \theta)$ can be obtained by dividing the total kinetic energy by half of the square of the velocity at that location. ${ }^{(16)}$ Thus, the equivalent mass on the circumference of the disk yields 


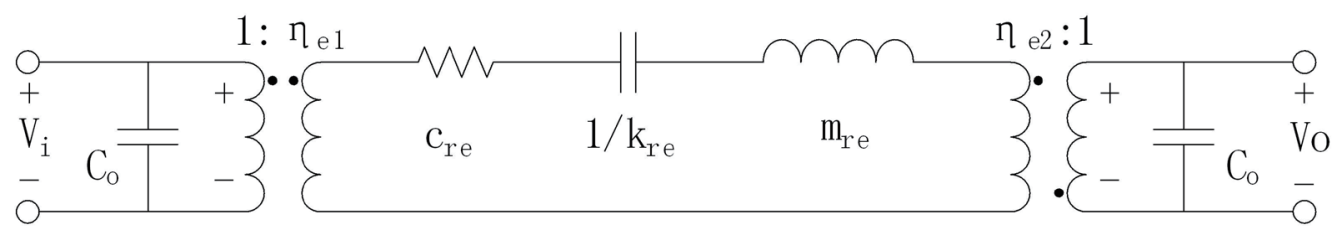

Fig. 3. (Color online) Physically consistent model using mass and stiffness for elements.

$$
m_{r e}=\frac{K E_{\text {tot }}}{\frac{1}{2} v^{2}\left(R_{\text {disk }}, \theta\right)}=\frac{2 \pi \rho t \int_{0}^{R_{\text {disk }}} r J_{1}^{2}(h r) d r}{J_{1}^{2}\left(h R_{\text {disk }}\right)},
$$

where $K E_{\text {tot }}$ is the kinetic energy of the disk and $v(r, \theta)$ is the velocity magnitude.

From Eq. (8), expressions for the equivalent stiffness and damping at a location on the disk can be obtained using the relations

$$
\begin{gathered}
k_{r e}=\omega_{0}^{2} m_{r e}, \\
c_{r e}=\frac{\omega_{0} m_{r e}}{Q}=\frac{\sqrt{k_{r e} m_{r e}}}{Q} .
\end{gathered}
$$

An expression for the electromechanical coupling factor at port $n$ can be obtained as

$$
\eta_{e n}=V_{p}\left(\frac{\partial C_{n}}{\partial x}\right), n=1,2
$$

The quantity $C_{0}$ in Fig. 3 represents the capacitance from an $\mathrm{I} / \mathrm{O}$ electrode to the $\mathrm{AC}$ ground, and as such, is primarily composed of combinations of electrode-to-resonator overlap capacitance and electrode-to-substrate capacitance.

\section{Extensional-mode Coupling Beams}

\subsection{Ideal model}

Because a radial-contour-mode disk vibrates in the radial direction, the required coupler modes are of the extensional type. The characteristics of a thin coupling beam (as a spring) are similar to those of an electrical transmission line, as shown in Fig. 4.

Hence, the extensional-mode beam vibration can be described by the following transmission (ABCD) matrix form, ${ }^{(16,17)}$ 


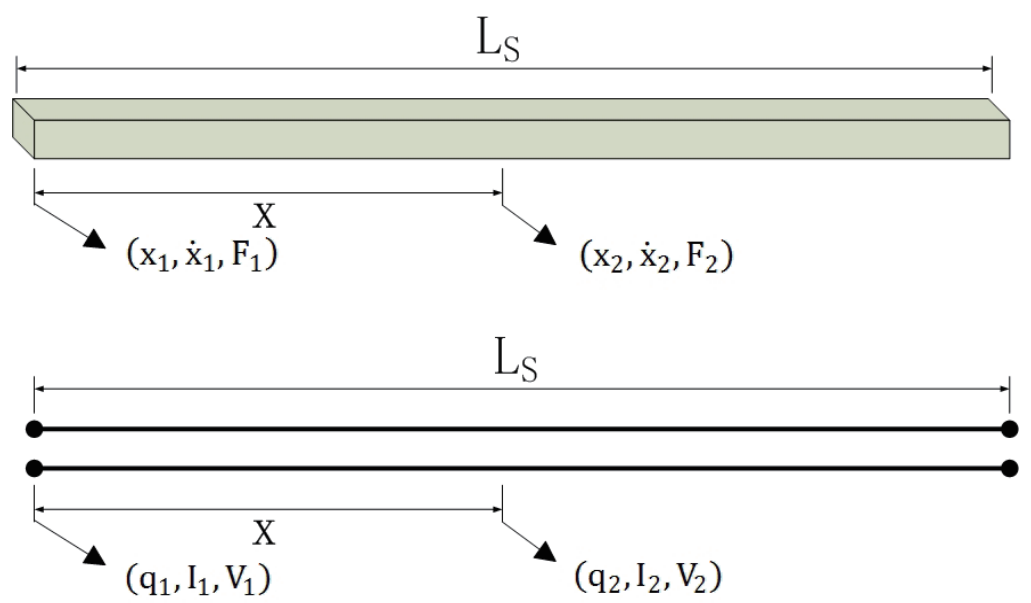

Fig. 4. (Color online) Transmission line models: (a) mechanical and (b) electrical.

$$
\left[\begin{array}{c}
F_{1} \\
\dot{x}_{1}
\end{array}\right]=\left[\begin{array}{cc}
\cos \beta x & j Z_{0} \sin \beta x \\
j \sin \beta x / Z_{0} & \cos \beta x
\end{array}\right]\left[\begin{array}{c}
F_{2} \\
\dot{x}_{2}
\end{array}\right],
$$

where $F$ and $x$ denote the force and displacement, $A_{C}$ and $L_{S}$ represent the cross-sectional area and length of the beam, respectively, $Z_{0}=A_{C} \sqrt{\rho E}=\sqrt{m k}, v_{p}=\sqrt{E / \rho}=\sqrt{k / m}, \beta=\omega / v_{p}$, and $\beta=\omega / V_{p}$.

Then, the $Z$-parameters of the general coupling beam model (i.e., the T network shown in Fig. 5) can be calculated from the ABCD parameters in Eq. (12) as

$$
\begin{gathered}
Z_{a}=\frac{A-1}{C}=-j Z_{0} \frac{\cos \beta L_{s}-1}{\sin \beta L_{s}}, \\
Z_{b}=\frac{D-1}{C}=-j Z_{0} \frac{\cos \beta L_{s}-1}{\sin \beta L_{s}}, \\
Z_{c}=\frac{1}{C}=-j Z_{0} \frac{1}{\sin \beta L_{s}} .
\end{gathered}
$$

When modeling an electrical equivalent circuit, the impedances $Z_{a}, Z_{b}$, and $Z_{c}$ can be replaced with capacitors or inductors as follows:

$$
Z=j \omega L \text { or } Z=\frac{1}{j \omega C}
$$




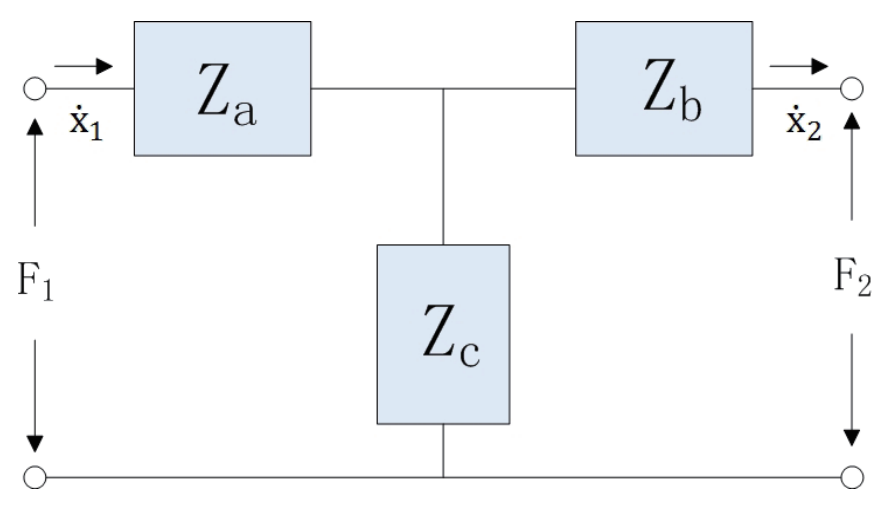

Fig. 5. (Color online) General coupling beam model.

\subsection{Coupling beam model under cross-sectional area variation}

Typically, resonators are fabricated in the same process and thus their material properties are generally consistent. As a consequence, the centroid offset of the coupling beam is often caused by the dimension deviation of the beam instead of material density variation. In general, the cross-sectional area $A_{C}$ in the transmission matrix (12) can be considered as a function of position $x$, i.e., $A c(x)$. By dividing the beam into $N$ equal segments (i.e., each segment has a length of $L_{S} / N$ ), the transmission matrix for each section is then given as

$$
\left[\begin{array}{c}
F_{1} \\
\dot{x}_{1}
\end{array}\right]=\left[\begin{array}{cc}
\cos \left(\beta \frac{L_{S}}{N}\right) & j Z_{0}(x) \cdot \sin \left(\beta \frac{L_{S}}{N}\right) \\
j \sin \left(\beta \frac{L_{S}}{N}\right) / Z_{0}(x) & \cos \left(\beta \frac{L_{S}}{N}\right)
\end{array}\right]\left[\begin{array}{c}
F_{2} \\
\dot{x}_{2}
\end{array}\right],
$$

where $Z_{0}(x)=A_{C}(x) \cdot \sqrt{\rho E}$. From the characteristics of the transmission matrix, the matrix of the entire beam can be obtained as follows by cascading each sectional matrix equation in the beam:

$$
\left[\begin{array}{ll}
A & B \\
C & D
\end{array}\right]=\prod_{x=1}^{x \rightarrow N, N \rightarrow \infty}\left[\begin{array}{cc}
\cos \left(\beta \frac{L_{S}}{N}\right) & j Z_{0}(x) \cdot \sin \left(\beta \frac{L_{S}}{N}\right) \\
j \sin \left(\beta \frac{L_{S}}{N}\right) / Z_{0}(x) & \cos \left(\beta \frac{L_{S}}{N}\right)
\end{array}\right],
$$

where $N$ is a sufficiently large integer such that the actual beam shape can be well approximated. Theoretically, it tends to be infinite.

In the absence of research about how the beam centroid actually becomes offset due to the process variations, and considering that the beam usually has a high aspect ratio and is fabricated by some manufacturing technology, ${ }^{(18,19)}$ which leads to inclination effects, ${ }^{(20,21)}$ in the actual beam, a trapezoidal beam is utilized here for simplification, as shown in Fig. 6 . 


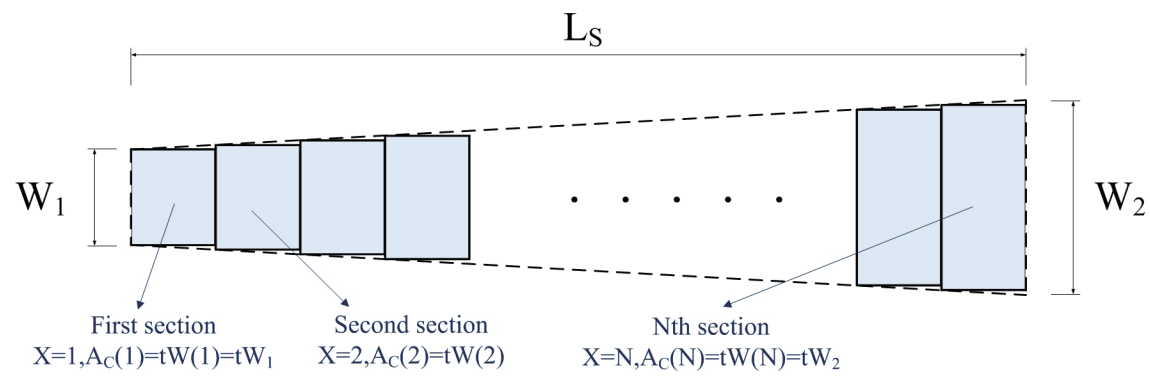

Fig. 6. (Color online) Top view of the trapezoidal coupling beam model (with thickness $t$ ).

Specifically, the cross-sectional area $A_{C}(x)$ of the coupling beam is set as a linear function of the beam length as follows:

$$
A_{C}(x)=t \cdot\left(\frac{W_{2}-W_{1}}{N-1} x+\frac{N W_{1}-W_{2}}{N-1}\right)
$$

where $W_{1}$ and $W_{2}$ denote the beam widths at the two ends as shown in Fig. 6.

\section{Coupled Double-disk Resonator}

\subsection{Construction}

Without loss of generality, consider a coupled double-disk resonator as an example of an array composite resonator, as shown in Fig. 7, which consists of two single-disk resonators connected through a coupling beam. The two single-disk resonators are of the same type as the resonator shown in Fig. 1. Moreover, the two single-disk resonators use the same input source $v_{i}$ and share the same bias voltage $V_{P}$, while the output electrodes are connected in parallel in order to increase the output current and thus reduce the dynamic impedance of the resonator linearly.

To make the single-disk resonators resonate at the same frequency with the same amplitude and phase, the dimension of the coupling beam must be adjusted to make it act as a spring with infinite effective stiffness. ${ }^{(22,23)}$ Specifically, it must satisfy the following conditions: $\beta L_{S}=(2 n+1) \pi, n=0,1,2,3, \ldots, Z_{a}=Z_{b}=-2 Z_{c}$. That is,

$$
\begin{gathered}
Z_{a}=\frac{A-1}{C}=\frac{j 2 Z_{0}}{\infty}, \\
Z_{b}=\frac{D-1}{C}=\frac{j 2 Z_{0}}{\infty}, \\
Z_{c}=\frac{1}{C}=\frac{-j Z_{0}}{\infty},
\end{gathered}
$$




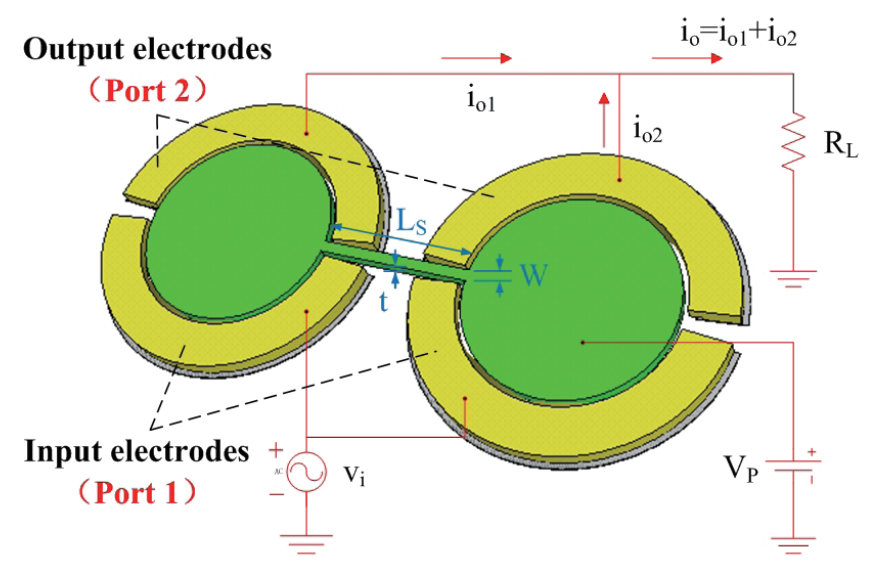

Fig. 7. (Color online) Coupled double-disk resonator $\left(L_{S}=\lambda / 2\right.$, where $\lambda$ is the corresponding wavelength of the resonant frequency of the resonator).

where the modulus of each impedance tends to positive infinity. Furthermore, the coupling beam length is calculated as follows:

$$
L_{S}=\frac{2 n+1}{\pi} \cdot \frac{1}{f_{0}} \cdot \sqrt{\frac{E}{\rho}}, n=0,1,2,3 \ldots
$$

where $f_{0}$ is the resonant frequency and also the operating frequency. When the length of the coupling beam is equal to an odd multiple of the half-wavelength (and normally takes a halfwavelength), the entire array-composite resonator theoretically behaves similarly to each of its constituent single-disk resonators, but with lower impedance, better linearity, and better power handling capability. ${ }^{(9)}$

By following the guidelines on how to select the length of the coupling beam on the basis of Eq. (23), the desired array-composite resonator can be established with the coupled double-disk resonator in Fig. 7. Specifically, the length of the beam is set to $\lambda / 2$, where $\lambda$ is the wavelength corresponding to the operating frequency of the resonator. To make the result more intuitive, the mode shape of the coupled double-disk resonator is shown in Fig. 8. By comparing with Fig. 2, one can observe in Fig. 8 that the resonant frequency and mode shape of the coupled resonator are unchanged.

\subsection{Equivalent circuit model}

Using the design method that Wang and Nguyen mentioned in Ref. 10, the equivalent circuit model of the coupled double-disk resonator can be established, as shown in Fig. 9, in which the circuit model of each single-disk resonator is connected to that of the coupling beam by a linear transformer ${ }^{(24)}$ with $\eta_{c n}=1: 1$ turn ratio, where $n=1$ and 2 denote the two electrodes.

Furthermore, by replacing the mechanical model in Fig. 7 with the circuit model in Fig. 9 and assuming that the dimension of the coupling beam has no deviation, the entire circuit is symmetrical, i.e., $i_{c a}=i_{c b}=i_{c c} / 2$. Thus, from Eqs. (20)-(23), the equivalent impedance at each 


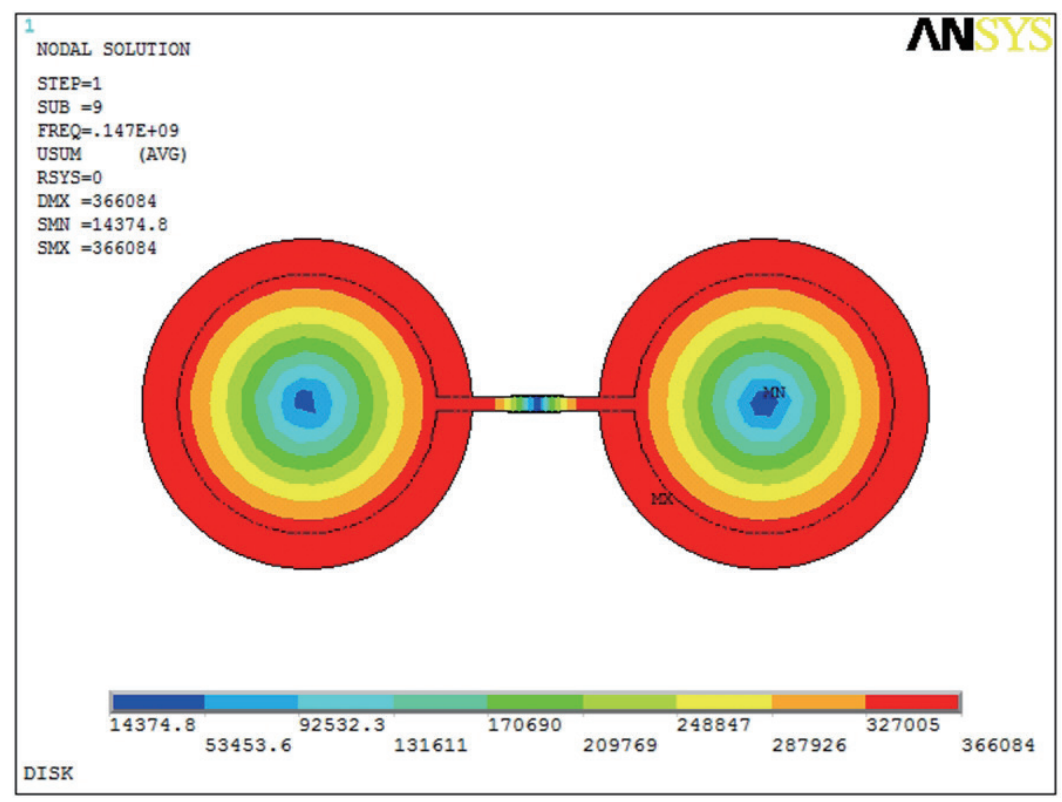

Fig. 8. (Color online) Mode shape of the ideal coupled double-disk resonator.

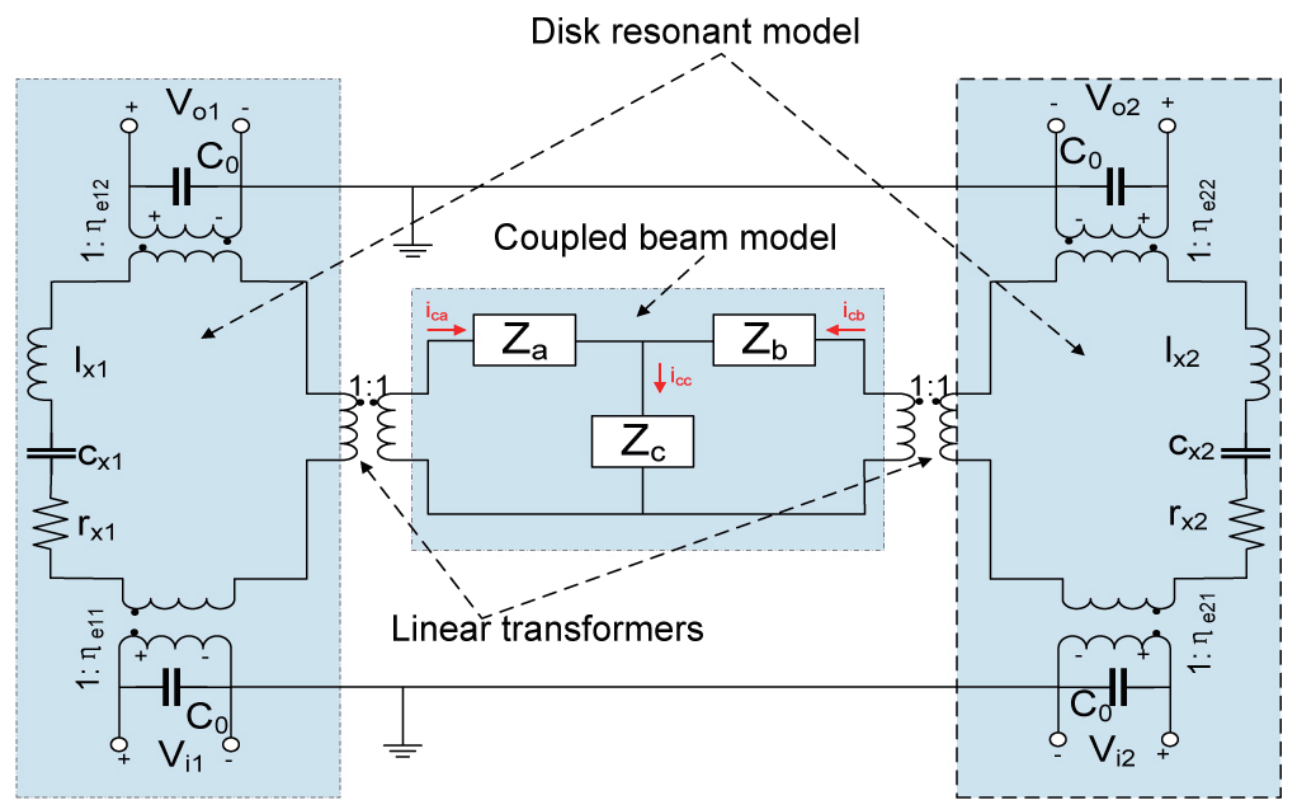

Fig. 9. (Color online) Equivalent circuit model of the coupled double-disk resonator.

end of the coupling beam from the disk resonator is zero when the length of the coupling beam is set to $\lambda / 2$, implying that the entire coupled double-disk resonator should behave similarly to one of its constituent single-disk resonators.

Owing to process variations, however, the centroid of the coupling beam may shift slightly, which causes the asymmetry of the circuit and ultimately affects the vibration of each disk. To study this problem, the model of the coupling beam with cross-sectional area variations in Sect. 3.2 is adopted and then a circuit simulation is performed using PSpice. 


\section{Results and Discussion}

The ideal coupled double-disk resonator has identical disks and an ideal coupling beam with its length equal to half the wavelength corresponding to the operating frequency. In this ideal case, the two disks resonate with the same amplitude and phase at the same frequency, and produce a consistent output current to reduce the equivalent dynamic impedance. In practice, however, various process variations in lithography, etching, and so forth lead to deviations from a perfect match between the array elements. As a result, the disk radius and the dimension of the beam in the array structure may have random distributions around the ideal design values. Herein, the performance of the coupled double-disk resonator is evaluated when the dimension of the coupling beam has some deviation. Specifically, the performance is mainly examined from three aspects: (1) the resonant frequency, (2) the current amplitude of each disk, and (3) $Q$.

To simulate the case of the centroid offset of the coupling beam, a trapezoid beam is adopted here [see Fig. 10(a)], in which the width at its left end is fixed at $W_{1}=2.00 \mu \mathrm{m}$ and that at its right end is denoted by a variable $W_{2}$ to model the trapezoid beam (i.e., the centroid offset of the beam). Specifically, $W_{2}$ takes a value from the list in Table 1. Ideally, when $W_{2}=W_{1}=2.00 \mu \mathrm{m}$, the coupling beam centroid has no offset; thus, it can be used as the reference for comparisons. For all other cases with $W_{2}>W_{1}$, the centroid of the beam shifts to the right. To clearly illustrate the actual beam centroid offset, the offset distances and the ratios of the offset distances to the beam length are both given in Table 1 .

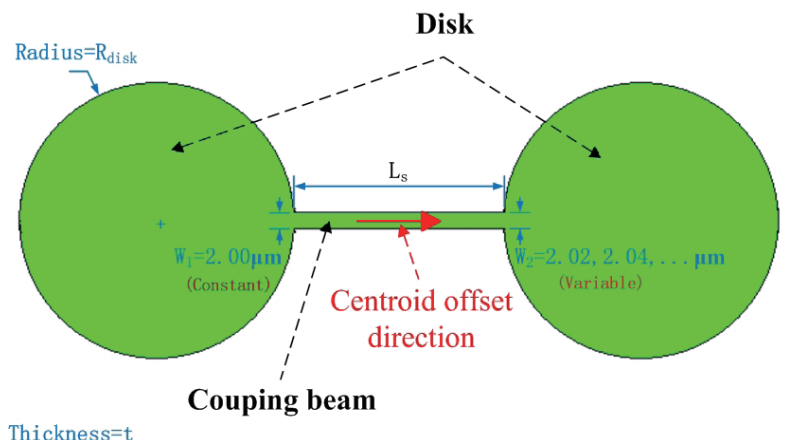

(a)

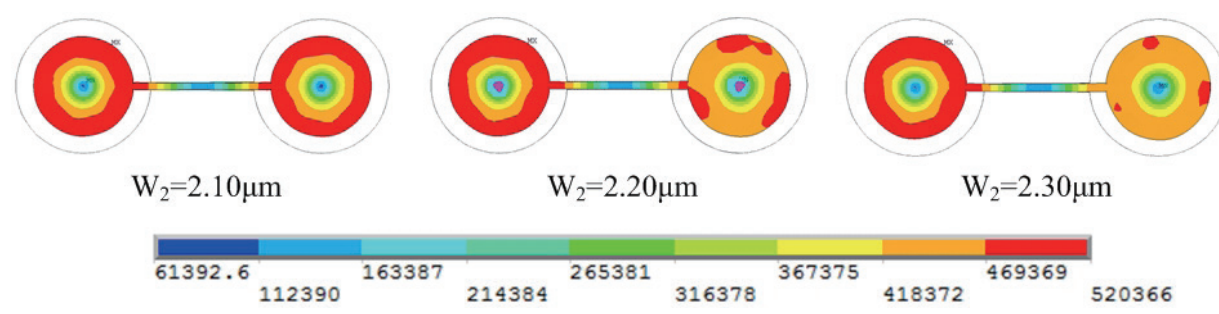

(b)

Fig. 10. (Color online) Coupling beam centroid offset model and diagram of partial mode shapes (colors represent degrees of deformation). 
Table 1

Centroid offsets.

\begin{tabular}{lcc}
\hline$W_{2}(\mu \mathrm{m})$ & Centroid offset $(\mu \mathrm{m})$ & Offset degree $(\%)$ \\
\hline 2.00 & +0 & +0 \\
2.02 & +0.0342 & +1.242 \\
2.04 & +0.0681 & +2.474 \\
2.06 & +0.1016 & +3.692 \\
2.08 & +0.1348 & +4.899 \\
2.10 & +0.1677 & +6.094 \\
2.20 & +0.3273 & +11.895 \\
2.30 & +0.4793 & +17.419
\end{tabular}

Note: offset degree $=$ centroid offset / LS; the symbol "+" means to the right.

Table 2

Resonator design parameters.

\begin{tabular}{lccc}
\hline Row No. & Parameters & Results & Unit \\
\hline 1 & Disk diameter, $2 R_{\text {disk }}$ & 36 & $\mu \mathrm{m}$ \\
2 & Disk and beam thickness, $t$ & 2.1 & $\mu \mathrm{m}$ \\
3 & Stem diameter, $2 R_{\text {stem }}$ & 2 & $\mu \mathrm{m}$ \\
4 & I/O electrode width, $W_{e}\left(\phi_{1}=\phi_{2}\right)$ & 53.4 & $\mu \mathrm{m}$ \\
5 & Electrode-to-resonator gap, $d_{0}$ & 87 & $\mathrm{~nm}$ \\
6 & Coupling beam length, $L_{S}$ & 27.5155 & $\mu \mathrm{m}$ \\
7 & Density, $\rho$ & 2300 & $\mathrm{~kg} / \mathrm{m}^{3}$ \\
8 & Young's modulus, $E$ & 150 & $\mathrm{GPa}$ \\
9 & Poisson ratio, $\sigma$ & 0.226 & - \\
10 & Frequency scalar, $k$ & 0.342 & $\mathrm{MHz} / \mu \mathrm{m}$ \\
11 & Mode-dependent factor, $\alpha$ & 1 & - \\
12 & $h(6)$ & 0.115 & $\mu \mathrm{m}^{-1}$ \\
13 & Force/dependent ratio, $A$ & 0.0111 & $\mu \mathrm{m}$ \\
14 & AC input amplitude, $V_{i}$ & 1 & $\mathrm{~V}$ \\
15 & Biased voltage, $V_{P}$ & 6 & $\mathrm{~V}$ \\
16 & $\partial C_{n} / \partial r(2)$ & $1.3118 \mathrm{e}-07$ & $\mathrm{~F} / \mathrm{m}$ \\
17 & $F_{i}(1)$ & $7.8709 \mathrm{e}-07$ & $\mathrm{~N}$ \\
18 & $P_{i}(3)$ & 7.0189 & $\mathrm{KPa}$ \\
19 & Ideal Q-factor, $Q(5)$ & 10361.3 & - \\
20 & Resonator mass, $m_{r e}(8)$ & $3.8971 \mathrm{e}-12$ & $\mathrm{~kg}$ \\
21 & Resonator stiffness, $k_{r e}(9)$ & $3.3135 \mathrm{e}+06$ & $\mathrm{~N} / \mathrm{m}$ \\
22 & Damping factor, $c_{r e}(10)$ & $3.5935 \mathrm{e}-07$ & $\mathrm{~kg} / \mathrm{s}$ \\
23 & Coupling factor, $\eta_{e n}(11)$ & $7.9709 \mathrm{e}-07$ & $\mathrm{C} / \mathrm{m}$ \\
\hline & & &
\end{tabular}

Table 2 shows the design parameters of the resonator, including the dimensions of the resonator structure, the material parameters, and the associated model parameters, whereas the parameters of the circuit model of the coupling beam for the PSpice simulation are summarized in Table 3.

\subsection{Resonant frequency}

When the dimension of the coupling beam changes, the overall geometry of the resonant structure is affected accordingly, which may shift the resonant frequency of the resonator. 
Table 3

Electrical equivalent model of the coupling beam $(N=30)$.

\begin{tabular}{|c|c|c|c|c|c|c|c|c|c|}
\hline \multicolumn{10}{|c|}{ Width of right end of beam $\left(W_{2}\right)$ unit } \\
\hline Parameters & 2.00 & 2.02 & 2.04 & 2.06 & 2.08 & 2.10 & 2.20 & 2.30 & $\mu \mathrm{m}$ \\
\hline \multicolumn{10}{|c|}{ 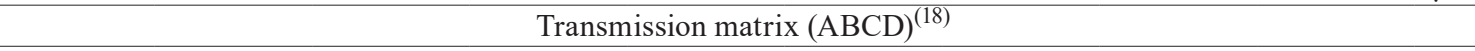 } \\
\hline$A$ & -1.000 & -0.994 & -0.989 & -0.984 & -0.979 & -0.974 & -0.950 & -0.927 & - \\
\hline$B$ & $(3.78 \mathrm{e}-20) \mathrm{i}$ & $(1.04 \mathrm{e}-09) \mathrm{i}$ & $(4.17 \mathrm{e}-09) \mathrm{i}$ & $(9.33 \mathrm{e}-09) \mathrm{i}$ & $(1.65 \mathrm{e}-08) \mathrm{i}$ & $(2.56 \mathrm{e}-08) \mathrm{i}$ & $(1.01 \mathrm{e}-07) \mathrm{i}$ & $(2.20 \mathrm{e}-07) \mathrm{i}$ & - \\
\hline$C$ & $(6.22 \mathrm{e}-12) \mathrm{i}$ & $-0.056 \mathrm{i}$ & $-0.223 \mathrm{i}$ & $-0.496 \mathrm{i}$ & $-0.870 \mathrm{i}$ & $-1.340 \mathrm{i}$ & $-4.994 \mathrm{i}$ & $-10.495 \mathrm{i}$ & - \\
\hline$D$ & $-1.000 \mathrm{i}$ & -1.005 & -1.010 & -1.015 & -1.021 & -1.026 & -1.052 & -1.077 & - \\
\hline \multicolumn{10}{|c|}{ Components of T network $^{(13)-(15)}$} \\
\hline$Z_{a}$ & $3.2 \mathrm{e} 11 \mathrm{i}$ & $-35.09 \mathrm{i}$ & $-8.88 \mathrm{i}$ & $-3.99 \mathrm{i}$ & $-2.27 \mathrm{i}$ & $-1.473 \mathrm{i}$ & $-0.390 \mathrm{i}$ & $-0.183 i$ & $\Omega$ \\
\hline$Z_{b}$ & $3.2 \mathrm{e} 11 \mathrm{i}$ & $-35.28 \mathrm{i}$ & $-8.97 \mathrm{i}$ & $-4.05 \mathrm{i}$ & $-2.32 \mathrm{i}$ & $-1.512 \mathrm{i}$ & $-0.410 \mathrm{i}$ & $-0.197 i$ & $\Omega$ \\
\hline$Z_{c}$ & $-1.6 \mathrm{e} 11 \mathrm{i}$ & $17.59 \mathrm{i}$ & $4.46 \mathrm{i}$ & $2.01 \mathrm{i}$ & $1.14 \mathrm{i}$ & $0.746 \mathrm{i}$ & $0.200 \mathrm{i}$ & $0.095 \mathrm{i}$ & $\Omega$ \\
\hline \multicolumn{10}{|c|}{ Equivalent components ${ }^{(16)}$} \\
\hline$C_{a}$ & - & $3.09 \mathrm{e}-11$ & $1.22 \mathrm{e}-10$ & $2.71 \mathrm{e}-10$ & $4.76 \mathrm{e}-10$ & $7.36 \mathrm{e}-10$ & $2.77 \mathrm{e}-09$ & $5.90 \mathrm{e}-09$ & $\mathrm{~F}$ \\
\hline$C_{b}$ & - & $3.07 \mathrm{e}-11$ & $1.20 \mathrm{e}-10$ & $2.67 \mathrm{e}-10$ & $4.66 \mathrm{e}-10$ & $7.17 \mathrm{e}-10$ & $2.63 \mathrm{e}-09$ & $5.47 \mathrm{e}-09$ & $\mathrm{~F}$ \\
\hline$L_{c}$ & - & $1.90 \mathrm{e}-08$ & $4.84 \mathrm{e}-09$ & $2.18 \mathrm{e}-09$ & $1.24 \mathrm{e}-09$ & $8.09 \mathrm{e}-10$ & $2.17 \mathrm{e}-10$ & $1.03 \mathrm{e}-10$ & $\mathrm{H}$ \\
\hline
\end{tabular}

Figure 11 shows the calculated resonant frequencies obtained from the ANSYS and PSpice simulations versus the centroid offset. One can observe that both curves give the same trend: when the width $W_{2}$ at the right end of the coupling beam increases (i.e., the centroid shifts to the right), the resonant frequency of the resonator increases.

Nevertheless, one can see that there is some discrepancy between the simulation results from ANSYS and PSpice. Specifically, the relative difference between the two simulation results increases as the coupling beam offset increases. Herein, the ANSYS simulation results are taken as the standard reference since the PSpice circuit model ignores the change in resonant frequency to simplify the calculations. In particular, the parameters of the circuit models of the disk and coupling beam are strongly related to the resonant frequency, as indicated by Eqs. (8)(10) and (12). Nevertheless, the resonant frequency is still considered to be reasonable for such treatment in PSpice since the error is only on the order of one in a million as can be seen later.

As shown in Fig. 11(a), when the centroid offset is less than 6\%, the resonant frequency changes by $45.7 \mathrm{ppm}$. Such a deviation can be corrected by tuning the electric stiffness in accordance with Ref. 9, in which a tuning voltage excursion of $8 \mathrm{~V}$ from -3 to $5 \mathrm{~V}$ can provide a $25.4 \mathrm{ppm}$ change in resonant frequency. It is expected that further improvement in tuning voltage excursion may enable the accommodation of $45.7 \mathrm{ppm}$ deviation in resonant frequency. When the offset further increases to $12 \%$, there is a $110 \mathrm{ppm}$ change in resonant frequency, which is difficult to correct by electric stiffness tuning. To illustrate this argument, assume that the tuning method in Ref. 9 is linear, i.e., the correction rate is $3.175 \mathrm{ppm} / \mathrm{V}$. To correct a 110 ppm deviation in resonant frequency, a voltage range of no less than $34.6 \mathrm{~V}$ would be required. Certainly, this is still acceptable for some applications. In the case of $18 \%$ centroid offset, however, the derivation in resonant frequency is nearly $250 \mathrm{ppm}$, and thus a tuning voltage range of $78.7 \mathrm{~V}$ for performing correction would be required, which is hardly acceptable in most practical applications. Moreover, the practical tuning method can be more complicated, especially when the number of resonators used in the array is large. 
ANSYSResults

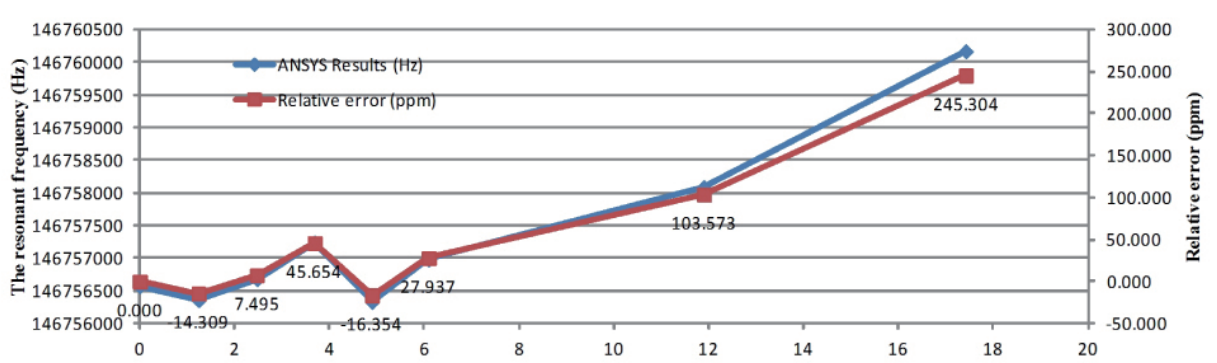

(a)

Pspice Results

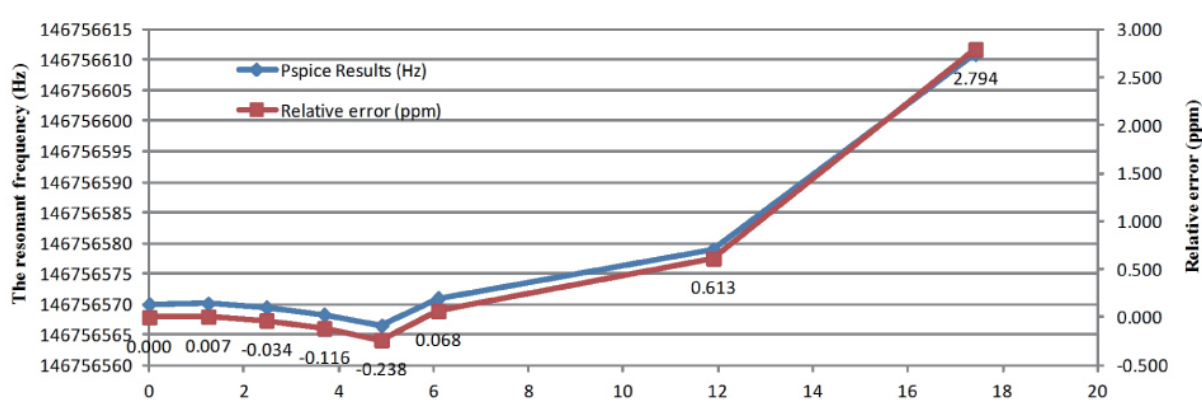

(b)

Fig. 11. (Color online) Resonant frequency versus centroid offset: (a) ANSYS and (b) PSpice simulations.

\subsection{Amplitude}

Random variations in the dimensions of resonators and coupling beams often compromise the amount that the output currents actually add, as mentioned in Ref. 9. Specifically, it was reported in Ref. 9 that a nine-disk array composite using three output resonators achieved only an output current of $2.78 \times$ (in contrast to ideally $3 \times$ ) that of a single-constituent resonator. According to Eq. (7), there are three factors that determine the output power, i.e., the bias voltage $V_{P}$, the change in $\partial C_{2} / \partial r$ in electrode-to-resonator overlap capacitance per unit radial displacement at the output port, and the disk amplitude. For the first two factors, the voltage $V_{P}$ is shared by all the disks and introduces no error, while the term $\partial C_{2} / \partial r$, which depends on the channel width between the disk and the electrode, is mainly related to the radius of the disk and has already been studied in Ref. 9. Herein, the effect of the disk amplitude on the output current is studied.

By ANSYS simulation, the disk amplitudes corresponding to various centroid deviations of the coupling beam are obtained and then converted into the output currents through Eq. (7). On the other hand, the output currents can also be obtained directly by PSpice simulation. Figures 12(a) and 12(b) depict the output currents of the left and right disks, respectively, obtained from both the ANSYS and PSpice simulations, versus the centroid offset. From Figs. 12(a) and 12(b), one can observe that the two results from ANSYS and PSpice are very close and show the same trend. When the beam centroid shifts towards the right disk, the output current of the right disk 
(a)

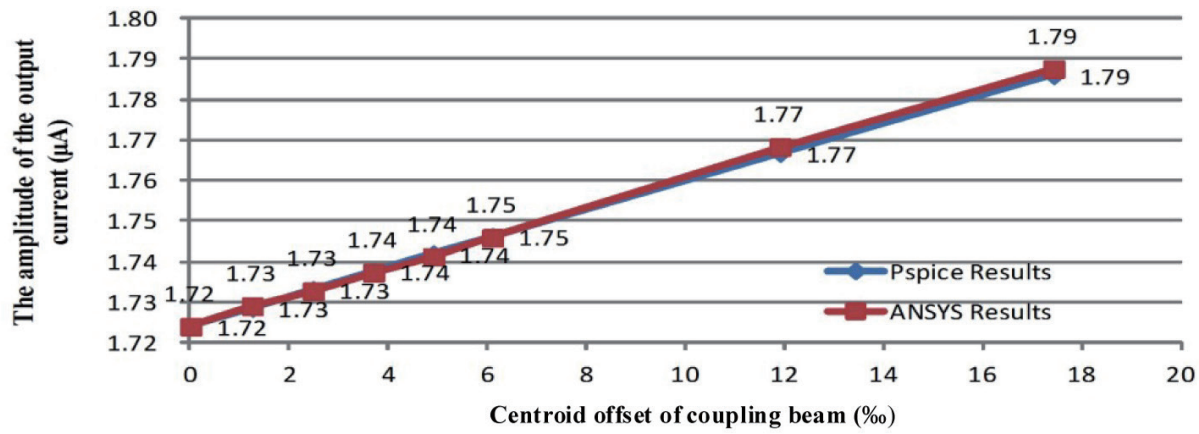

(b)
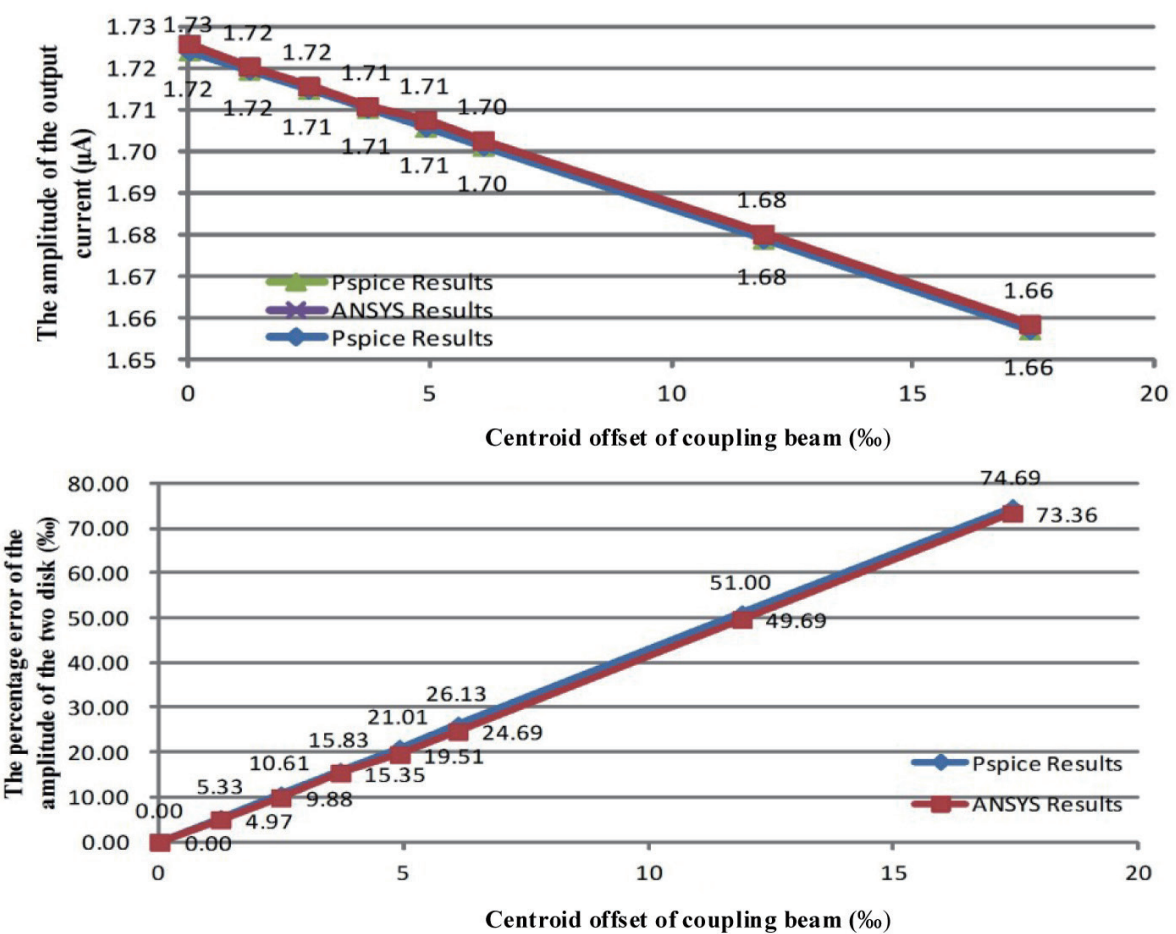

Fig. 12. (Color online) Amplitude versus centroid offset. (a) Amplitude of the left disk, (b) amplitude of the right disk, and (c) overall change.

decreases, while the output current of the left disk increases. Specifically, when the centroid shifts towards the right with offset $18 \%$, the output current amplitude of the left disk increases by $35.9 \%$ to reach $1.785 \mu \mathrm{A}$ (in reference to $1.724 \mu \mathrm{A}$ at zero offset), while the output current amplitude of the right disk decreases by $38.7 \%$ to $1.657 \mu \mathrm{A}$. Moreover, all these changes are nearly linear versus the centroid offset.

On the basis of the concept of equivalent mass, the results can be explained as follows. When the beam centroid shifts to the right, the equivalent mass of the right disk increases. If the total kinetic energy does not change, Eq. (8) implies that the velocity of the right disk decreases, and thus the amplitude of the right disk also decreases. In contrast, the equivalent mass of the left disk decreases, leading to increases in both the velocity and amplitude of the left disk. Comparing the mode shapes in Figs. 8 and Fig. 10(b), one can conclude that, when the coupling beam centroid shifts, the amplitude of one disk increases, while that of the other disk decreases. 
Note that the deviation can become more severe when there are more resonators used in the array. According to Ref. 9, the average amplitude of the disks decreases by nearly $85 \%$ for array-composite resonators with a $1 \%$ worst-case dimensional mismatch between the array elements when the number of resonators increases to seven. The relative mismatch between the left and right disks can be defined as the quotient of the difference between the amplitudes of the left and right disks divided by the disk amplitude under zero offset. Figure 12(c) shows the relative mismatch between the left and right disks obtained from the ANSYS and PSpice simulations versus the coupling beam centroid offset.

\subsection{Quality factor}

The quality factor is an important parameter in measuring the performance of a resonator. A high quality factor of a resonator implies a high frequency selectivity, good short-term stability, and a relatively low dynamic impedance. Therefore, it is necessary to study the quality factor variation caused by the centroid offset of the coupling beam. In this subsection, the PSpice simulation of the equivalent circuit of the resonator is utilized to obtain the spectral results, and then the corresponding quality factor is calculated as

$$
Q=\frac{f_{0}}{B W_{3 d B}}=\frac{f_{0}}{f_{2}-f_{1}}
$$

where $f_{0}$ is the resonant frequency of the resonator, $B W_{3 \mathrm{~dB}}$ is the $3 \mathrm{~dB}$ bandwidth, and $f_{2}$ and $f_{1}$ are the upper and lower bound frequencies of the $3 \mathrm{~dB}$ bandwidth, respectively.

Figure 13 shows the quality factor obtained from the PSpice simulation versus the coupling beam centroid offset. Obviously, when the dimension of the coupling beam is not changed (i.e., $W_{2}=2.00 \mu \mathrm{m}$ ), the corresponding quality factor is highest (i.e., $Q=10361.3$ ); here, the coupling beam does not contribute to the energy loss of the resonator. However, when the centroid shifts, the overall quality factor of the resonators decreases. From Fig. 13, one can see that severe performance degradation occurs when the centroid shifts to the right with an offset degree of $18 \%$, resulting in a decrease of nearly $19 \%$ in quality factor.

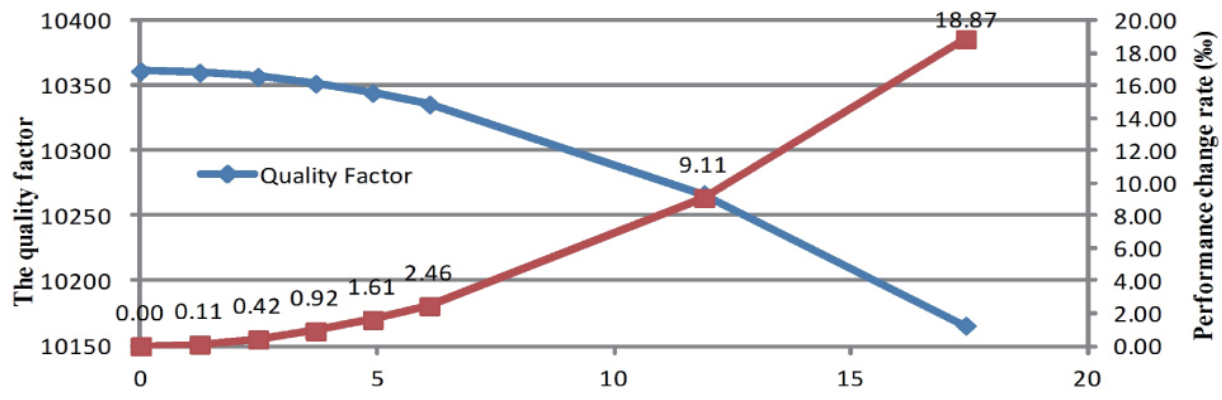

Fig. 13. (Color online) Quality factor versus centroid offset. 


\section{Conclusions}

The performance variations of an array composite resonator and the coupling beam centroid offset caused by process variations have been studied. Firstly, an equivalent circuit model for the trapezoid coupling beam was deduced to model the beam under the centroid offset. Then, a model for a double-disk coupled resonator including the beam centroid offset was established by combining the equivalent circuit model with the existing circuit model of the radial-contour mode disk resonator. Finally, numerical results were obtained by simulations using ANSYS and PSpice with the mechanical and electrical models, respectively.

It has been shown that the resonant frequency varied by 45.7, 110, and $250 \mathrm{ppm}$ when the beam centroid shifted by 6,12 , and $18 \%$, respectively. When the centroid shifted towards one disk by $18 \%$, the output current amplitude of this disk decreased by $38.7 \%$ o to $1.657 \mu \mathrm{A}$ (compared with $1.724 \mu \mathrm{A}$ at zero offset), while the output current amplitude of the other disk increased by $35.9 \%$ to reach $1.785 \mu \mathrm{A}$. In addition, when there was an offset, the $Q$ of the resonator decreased. In particular, when the centroid had an $18 \%$ offset, $Q$ decreased by nearly $19 \%$.

When there are more resonators in the array, a small deviation can be amplified and severely affects the performance of the array-composite resonator. The aim of this work is to provide resonator designers with a detailed understanding of the effects of the coupling beam centroid offset so that the performance of multidisk array-composite resonators can be maximized.

\section{Acknowledgments}

The authors acknowledge the support of this research by the National Natural Science Foundation of China under Grant Nos. 61871167 and 61376117 and the Key Research and Development Plan Project of Zhejiang Province under Grant No. 2018C01036.

\section{References}

1 R. C. Ruby, P. Bradley, Y. Oshmyansky, A. Chien, and J. D. Larson III: Proc. IEEE Ultrasonics Symp. (IEEE, 2001) 813-821.

2 G. Piazza: J. Vac. Sci. Technol. A 27 (2009) 776

3 Y. W. Lin, S. S. Li, Z. Ren, and C. T.-C. Nguyen: Proc. 2005 Electron Devices Meeting (IEDM) Tech. Dig. (IEEE, 2005) 4.

4 S.-S. Li, Y.-W. Lin, Y. Xie, Z. Ren, and C. T.-C. Nguyen: Proc. 17th IEEE Int. Conf. MEMS, 2004, Tech. Dig. (IEEE, 2004) 821-824.

5 C. T.-C. Nguyen: Proc. 26th Symp. Sensors, Micromachines App. Sys. (IEEE, 2009) 1-5.

6 Y. W. Lin, S. Lee, S. S. Li, Y. Xie, Z. Ren, and C. T.-C. Nguyen: IEEE J. Solid-State Circuits 39 (2004) 2477.

7 M. Baghelani and H. B. Ghavifekr: Proc. 2009 European Conf. Circuit Theory and Design (ECCTD) (IEEE 2009) 23-27.

8 M. U. Demirci and C. T.-C. Nguyen: J. Microelectromech. Syst. 15 (2006) 1419.

9 M. Akgul, Z. Ren, and C. T.-C. Nguyen: Proc. 2011 Joint Conf. IEEE Int. Frequency Control and the European Frequency and Time Forum (IEEE, 2011) 1-6.

10 K. Wang and C. T.-C. Nguyen: J. Microelectromech. Syst. 8 (1999) 534.

11 F. D. Bannon, III, J. R. Clark, and C. T.-C. Nguyen: IEEE J. Solid-State Circuits 35 (2000) 512.

12 C. T.-C. Nguyen: Ph.D. dissertation, Department of Electrical Engineering and Computer Sciences, University of California at Berkeley (1994)

13 M. Onoe: J. Acoust. Soc. Am. 28 (1956) 1158. 
14 J. Wang, Z. Ren, and C. T.-C. Nguyen: IEEE Trans. Ultrason. Ferroelectr. Freq. Control 51 (2004 1607.

15 M. M. Shalaby, M. A. Abdelmoneum, and K. Saitou: IEEE Trans. Ind. Electron. 56 (2009) 1022-1-30.

16 R. A. Johnson: Mechanical Filters in Electronics (Wiley, New York, 1983).

17 J. Basu, T. Kanti, R. R. Chaudhuri, and A. L. Roy: Proc. 2011 IEEE Applied Electromagnetics Conf. (AEMC) (IEEE 2011) 1-4.

18 I. Misri, P. Hareesh, S. Yang, and D. L. DeVoe: J. Micromech. Microeng. 22 (2012) 085017.

19 P. Hareesh, I. Misri, S. Yang, and D. L. DeVoe: J. Microelectromech. Syst. 21 (2012) 1513.

20 Z.-Y. Zhong, W.-M. Zhang, G. Meng, and J. Wu: J. Microelectromech. Syst. 22 (2013) 865.

21 F. E. H. Tay, J. Xu, Y. C. Liang, V. J. Logeeswaran, and Y. F. Yao: J. Micromech. Microeng. 121 (1999) 283.

22 Y. W. Lin, S. S. Li, Z. Ren, and C. T.-C. Nguyen: Proc. 2005 Electron Devices Meeting (IEDM) Tech. Dig. (IEEE, 2005) 4.

23 J. R. Clark, W.-T. Hsu, M. A. Abdelmoneum, and C. T.-C. Nguyen: J. Microelectromech. Syst. 14 (2005) 1298.

24 H. A. C. Tilmans: J. Micromech. Microeng. 6 (1996) 157. 